\title{
Prognostication of Electrocardiographic Changes With Status Epilepticus in African American Population
}

\author{
Mohankumar Kurukumbi ${ }^{\mathrm{a}, \mathrm{c}}$, Noha Solieman ${ }^{\mathrm{a}}$, Suzanne Al-Hamad ${ }^{\mathrm{a}}$, Siddartha Bhandary ${ }^{\mathrm{b}}$, \\ Nadia Yusuf ${ }^{\mathrm{a}}$, Annapurni Jayam-Trouth ${ }^{\mathrm{a}}$
}

\begin{abstract}
Background: Status epilepticus (SE) is a medical emergency and requires rapid diagnosis and treatment to reduce morbidity and mortality. The general approach to management and treatment of SE is largely unexplored and it remains unclear if other factors besides age and etiology represent independent outcome predictors for SE. There have been previous studies that document changing electrocardiogram (ECG) tracing through SE and following the termination of SE, indicating a dynamic, pathological response of the myocardium to this neurological process. Here we examine the ECG changes in African American patients during and after SE and consider the prognostic indication of ECG readings.
\end{abstract}

Methods: The study was conducted on 113 African American patients older than 15 years who experienced SE following new onset or pre-existing epilepsy. Data were compiled through a retrospective analysis of the type of ECG changes with comparison of ECGs taken within 6 hours and after 24 hours in patients with SE.

Results: ECG changes were documented in $81 \%$ of patients with SE in the first 6 hours. Of the eight patients who died, seven had persistent ECG abnormalities. Looking at our group of patients with new onset epilepsy, $25 \%$ returned to baseline function post SE, $46 \%$ were left with some disability and $19 \%$ resulted in death. This can be compared to patients who had a history of seizures in which $90 \%$ returned to baseline, $6 \%$ were left with a disability and $3 \%$ resulted in death.

Conclusions: The high mortality rate associated with SE patients having ECG abnormalities indicates that close observation of car-

Manuscript accepted for publication April 14, 2014

${ }^{a}$ Department of Neurology, Howard University Hospital, Washington, DC, USA

${ }^{\mathrm{b}}$ Department of Public Health, Yale School of Public Health, New Haven, Connecticut, USA

${ }^{\mathrm{c} C}$ Corresponding Author: Mohankumar Kurukumbi, Department of

Neurology, Howard University Hospital, 2041 Georgia Ave.,

Washington, DC, 20060, USA. Email: mohan311@gmail.com

doi: http://dx.doi.org/10.14740/jnr271w diac function is essential in managing SE. Knowing that persistent ECG changes following SE are predictive of increased morbidity and mortality gives us the information we need to begin to change the way we manage and monitor SE patients. Cardiac monitoring using ECGs is a cost effective and non-invasive method to allow for early detection of potentially life threatening cardiovascular abnormalities in patients during and after SE.

Keywords: Prognostication; Status epilepticus; ECG changes

\section{Introduction}

Status epilepticus (SE) is a medical emergency requiring prompt diagnosis and treatment to reduce significant morbidity and mortality. Although SE is more common in pediatric patients, mortality in adult age group is significantly larger than in the pediatric population $[1,2]$ and patients with new onset seizures after age 65 have a risk of SE equal to that of children [3]. In the general approach to SE, the optimum sequence and timing of treatment is largely unexplored and it is not yet clear if other factors besides age and etiology represent independent outcome predictors of SE [4]. Here we examine the ECG changes in 113 African American patients during and after SE and consider the prognostic indication of ECG readings. ECGs are the most widely obtained cardiac test, and thus offer a readily available and inexpensive screen for myocardial abnormalities. SE treatment strategies vary substantially from one institution to another due to lack of data to support one treatment over another [5]. This information can be used to improve the treatment of patients with SE by the addition of ECG monitoring as well as suggesting the use of ECG findings when determining patient prognosis.

\section{Methods}

The study population was based on 150 patients with SE. Thirty-seven patients were excluded from the study because of unavailable ECGs after $24 \mathrm{~h}$ following onset of SE, and 113 were included. For clinical purposes, an operational 


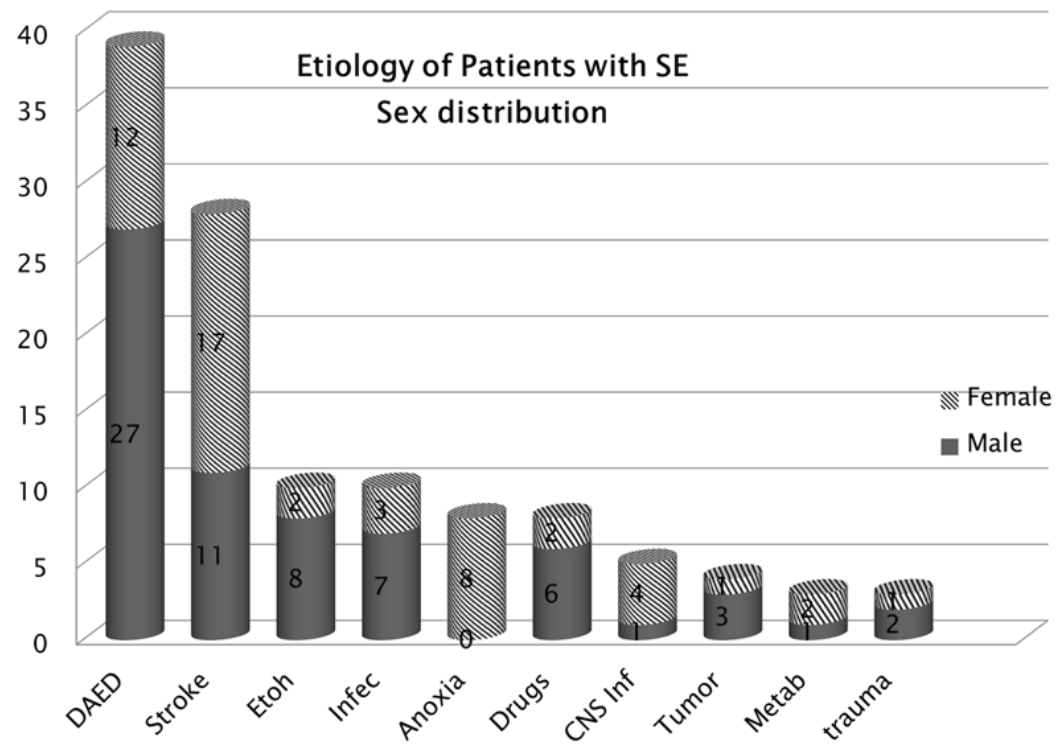

Figure 1. Etiology of SE according to sex. Strokes were the most common cause of SE in females while noncompliance was common in males.

definition of SE is 5 min of continuous seizures or discrete seizures with incomplete recovery of consciousness. This provides an appropriate time interval to initiate treatment for impending SE and prevent more prolonged or refractory SE. Because generalized tonic-clonic seizures usually last about $1 \mathrm{~min}$, longer seizure activity implies a failure of the physiologic factors that normally terminate a seizure. Data were compiled through a retrospective analysis of the type of ECG changes with comparison of ECGs taken within $6 \mathrm{~h}$ and after $24 \mathrm{~h}$ in patients with SE. Patients included were 113 African
American patients, age greater than 15 years, who were admitted to Howard University Hospital with SE from January 2007 to December 2011. Each patient had an ECG within $6 \mathrm{~h}$ from the onset of SE and greater than $24 \mathrm{~h}$ after seizure cessation. ECGs were initially classified by defining specific parameters of rate, QRS axis, PR and QT intervals, ST changes and voltage. Secondly, types of rhythm abnormalities, and their frequency of occurrence characterized ECGs. Finally, ECGs for each patient were reviewed for comparison between the ECGs obtained within $6 \mathrm{~h}$ from the onset of SE

\section{Ischemic Changes}

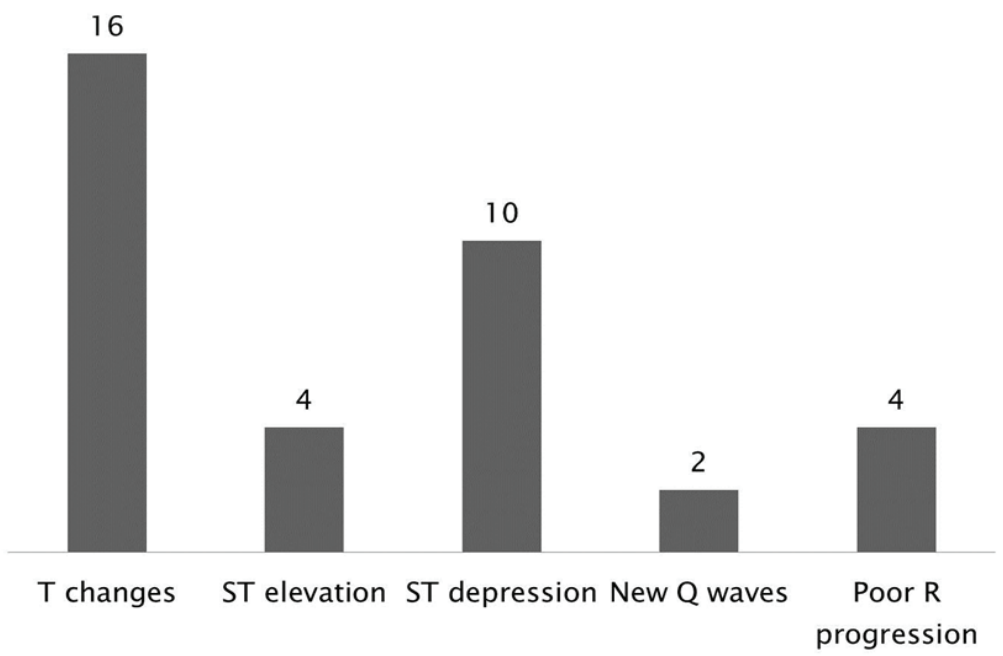

Figure 2. Breakdown of the ischemic changes following SE and it shows that the most common changes were $\mathrm{T}$ changes followed by ST depression. 


\section{Arrhythmia}

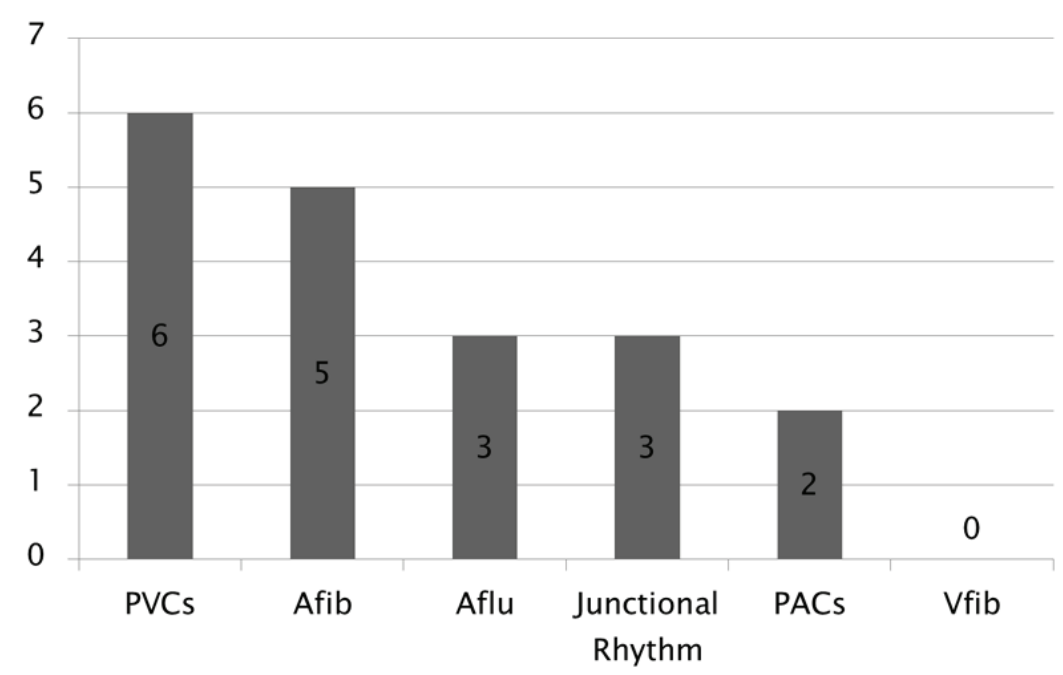

Figure 3. Type of arrhythmias observed following SE.

and those obtained greater than $24 \mathrm{~h}$ after the cessation of the seizure. All ECGs were interpreted by a faculty member of Howard University Hospital cardiology division.

\section{Results}

In our study population $77 \%$ of patients had a history of epilepsy while $23 \%$ of patients were having new onset seizure presenting as SE. Males were dominant in our study (58\%). Patient's age ranged from 16 to 83 years with a mean of 53.4 years. Our patients who experienced SE were dominated by individuals with generalized tonic-clonic seizures. In our study population, strokes were the most common cause of SE in females while noncompliance was the most common in males (Fig. 1). Anoxia was dominant in the female population. Phenytoin was the most common drug found to be used in patients with history of epilepsy as monotherapy $(66 \%)$ or in combination, and levetiracetam was the second most common drug used (20\%).

ECG changes were documented in $81 \%$ of patients with $\mathrm{SE}$ in the first $6 \mathrm{~h}$. The most frequently observed ECG abnormalities were ischemic patterns, conduction abnormalities and arrhythmia. Ischemia was observed in 19 males and 17 females, conduction abnormalities in 15 males and 13 females, arrhythmia in 14 males and five females, bradycardia in three females and no males, and voltage disturbances were observed in one male and one female. Arrhythmias were

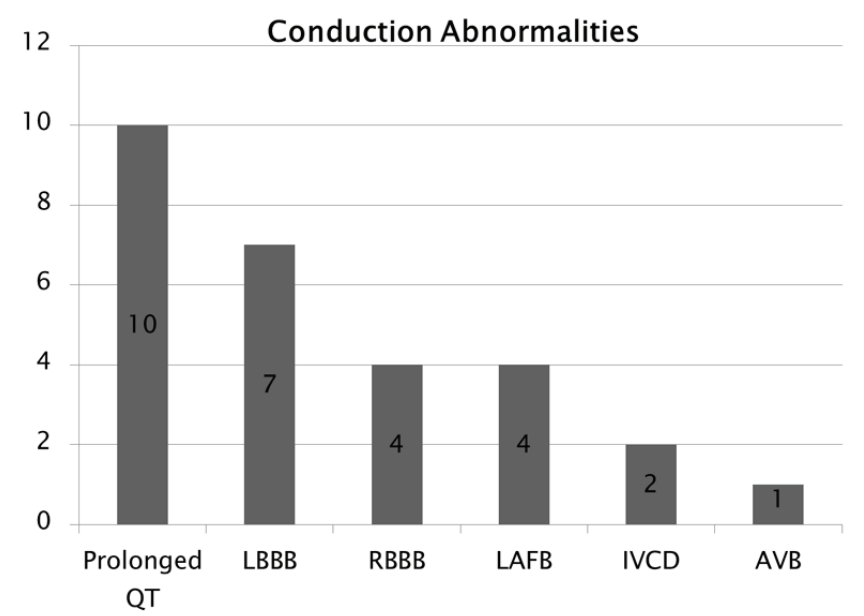

Figure 4. Conduction abnormalities. Note that prolonged QT was the cost common abnormality which can also be associated with phenytoin side effect. 


\section{Comparison of type of abnormalities}

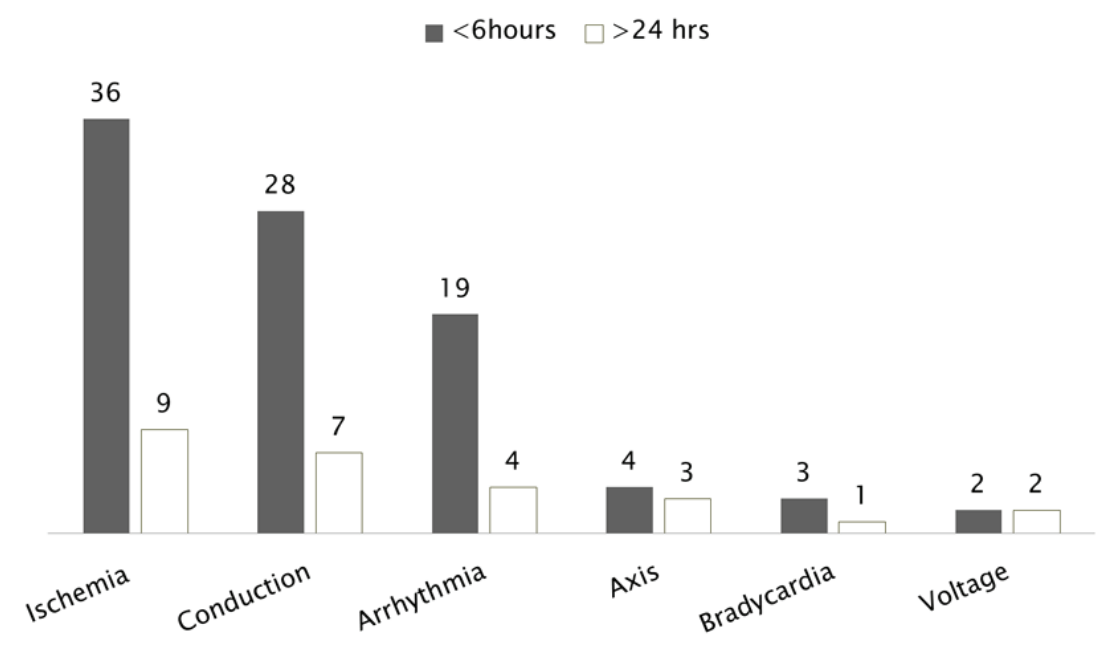

Figure 5. ECG abnormalities occurring during SE (within $6 \mathrm{~h}$ ) and more than $24 \mathrm{~h}$ after SE.

more frequently observed in males while bradycardia was more frequent in females.

The most common ischemic changes were $\mathrm{T}$ changes appreciated in 16 patients, followed by ST depression in 10 patients, ST elevation in four patients, poor R progression in four patients and new $\mathrm{Q}$ waves in two patients (Fig. 2).

The types of arrhythmias observed included premature ventricular contractions (PVCs) in six patients, atrial fibrillation (Afib) in five patients, atrial flutter (Aflu) in three patients, junctional rhythm in three patients and premature atrial contractions (PACs) in two patients (Fig. 3).

Figure 4 depicts the types of conduction abnormalities observed, including 10 patients with prolonged QT interval, seven patients with left bundle branch block (LBBB), four patients with right bundle branch block (RBBB), four patients with left anterior fascicular block (LAFB), two patients with intra-ventricular conduction delay (IVCD) and one patient with AV block (AVB). QT prolongation was the most common conduction abnormality which can be also associated with phenytoin's side effect.

Thirty-six patients were noted to have ischemia within the first $6 \mathrm{~h}$ following onset of SE, and this number dropped to nine patients who had persistent ischemic changes $24 \mathrm{~h}$ after onset of SE. A similar pattern was seen with conduc-

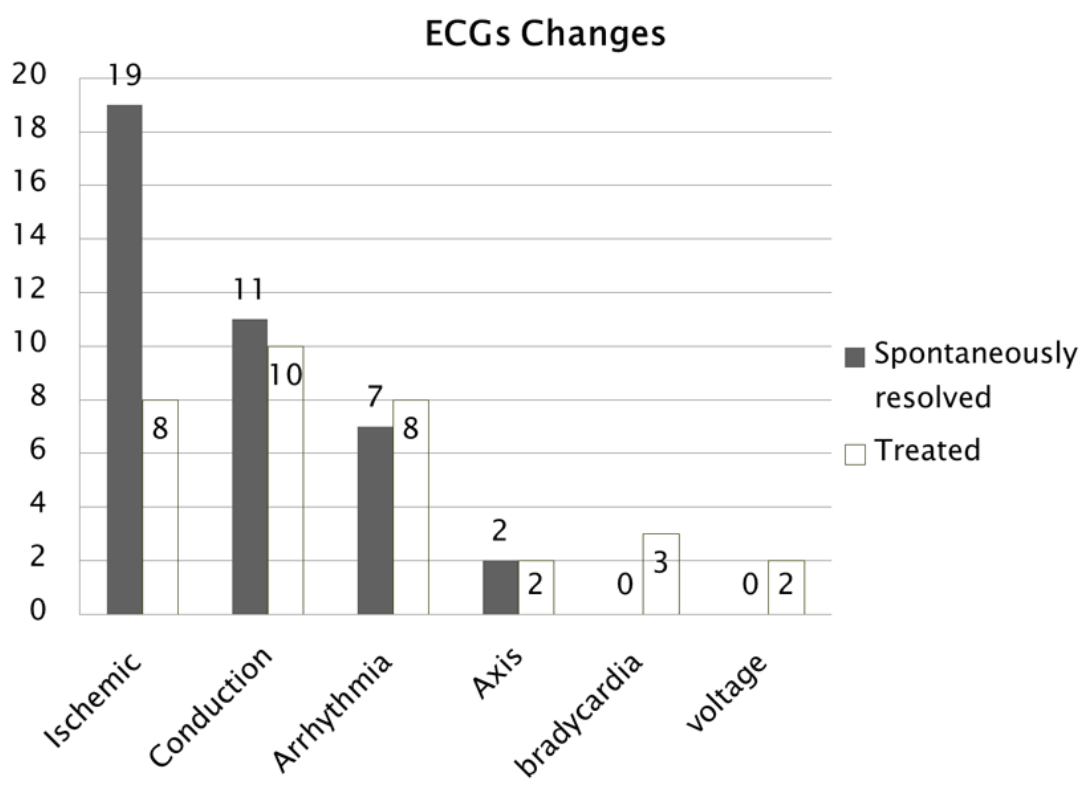

Figure 6. Comparison of ECG changes that occurred and either resolved spontaneously or needed treatment. 


\section{Type of persistent ECG changes associated with mortality (7)}

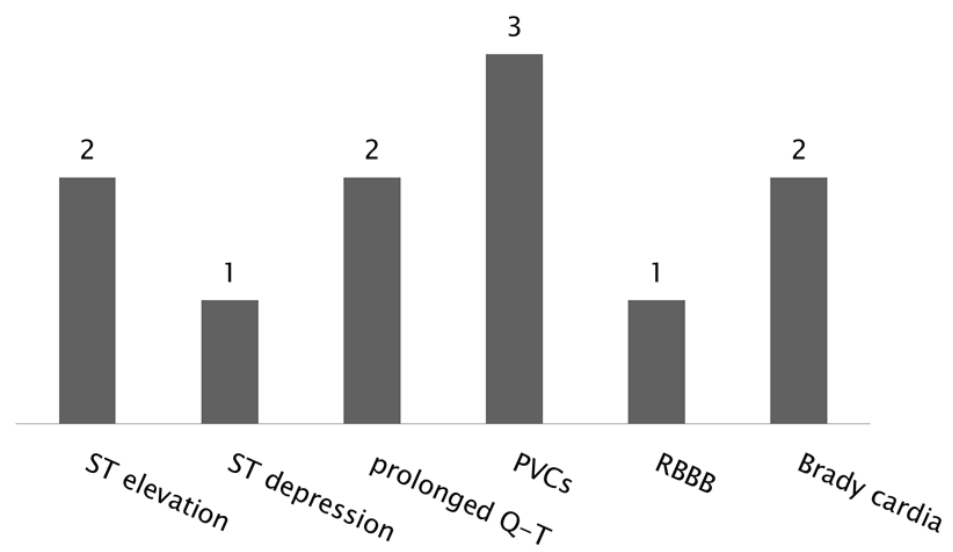

Figure 7. Types of persistent ECG abnormalities associated with mortality. There were seven patients with persistent ECG abnormalities who died. Some of these patients experienced more than one type of abnormality.

tion abnormalities dropping from 28 patients in the first $6 \mathrm{~h}$ to seven patients after $24 \mathrm{~h}$, and arrhythmia dropping from 19 patients to four patients after $24 \mathrm{~h}$. This comparison is presented in Figure 5.

Many ECG abnormalities resolved spontaneously and did not require therapy; however, potentially life threatening ECG abnormalities that did not spontaneously resolve within a reasonable length of time were treated and reversed with medications and pacemakers (Fig. 6). Eight patients with ischemic changes, 10 with conduction defects, eight with arrhythmias, three patients with bradycardia and two patients with voltage defects did not resolve spontaneously therefor requiring medical management.

Of the 95 patients $(84 \%)$ with ECG changes, 61 were persistent. Of the eight patients who died, seven had persis- tent ECG changes and one had ECG changes that did not persist beyond $24 \mathrm{~h}$. Seven out of the 61 patients with persistent ECG abnormalities died whereas one out of 34 patients with non-persistent ECG abnormalities died. Although the mortality of patients with persistent ECG changes was clinically apparent it was not statistically significant due to our small sample size $(\mathrm{P}<0.1220)$.

The types of persistent ECG abnormalities associated with mortality were three patients with PVCs, two with ST elevation, one with ST depression, two with prolonged QT, one with RBBB and two with bradycardia (Fig. 7). Some patients had more than one abnormality noted on their EKG. The cause of death (Fig. 8) was determined to be cardiac arrest in two patients, anoxic encephalopathy in two patients, myocardial infarction in three patients and subarachnoid

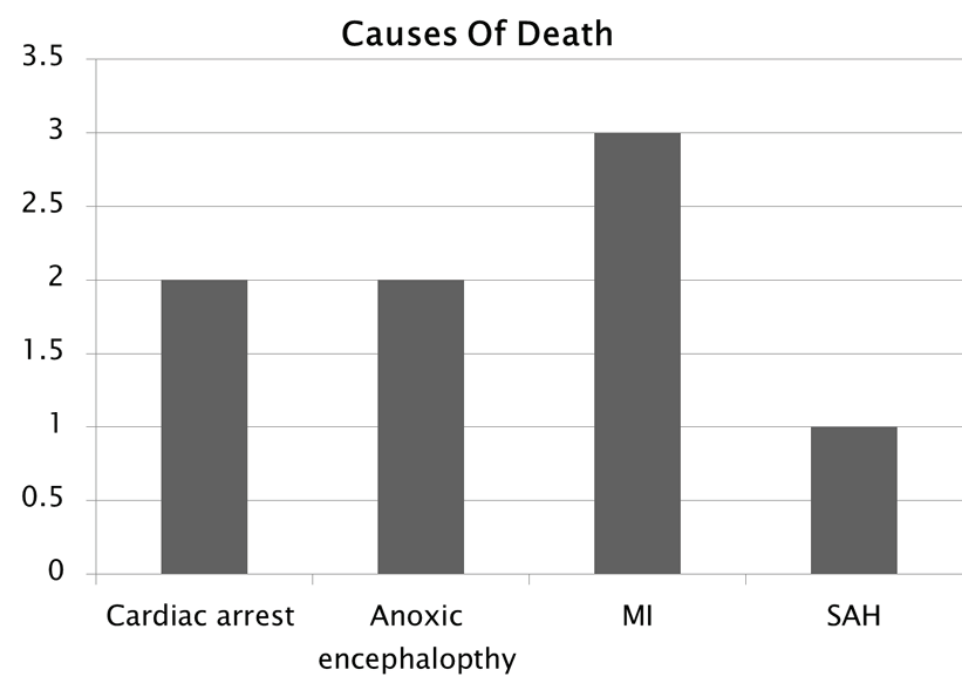

Figure 8. Determined cause of death. 


\section{Comparison of Patient Outcomes}

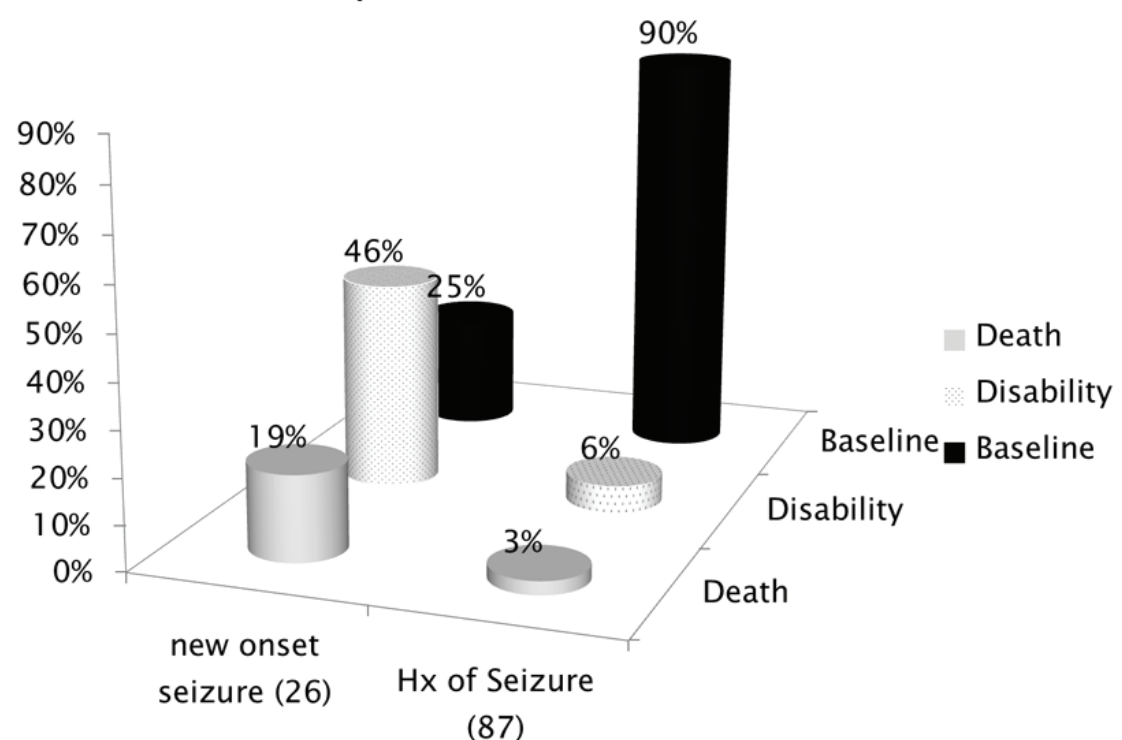

Figure 9. Patient outcomes comparing those with new onset epilepsy to those patients who had a history of epilepsy.

hemorrhage in one patient. Looking at our group of patients with new onset epilepsy, $25 \%$ returned to baseline function post SE, $46 \%$ were left with some disability and $19 \%$ resulted in death. This can be compared to patients who had a history of seizures in which $90 \%$ returned to baseline, $6 \%$ were left with a disability and 3\% resulted in death (Fig. 9).

\section{Discussion}

SE is clinically defined as 5 min or more of continuous clinical and/or electrographic seizure activity or recurrent seizure activity without recovery to baseline between seizures [5]. $\mathrm{SE}$ is a medical emergency and requires prompt evaluation and treatment. Although SE occurs in all age groups, it has a bimodal distribution across the lifespan [6]. It is most common among children younger than 1 year of age and adults older than 60 years. Older adults are at the highest risk of developing SE, with an incidence of 86 per 100,000 annually. The risk of SE occurrence in older adults is three to ten times the risk compared to younger adults $[1,7,8]$. Most studies document that about $40 \%$ of patients with SE have a history of epilepsy. It is most common in the setting of partial-onset epilepsy [9]. The goal of therapy is rapid termination of clinical and electrical seizure activity; the longer a seizure continues, the greater the likelihood of an adverse outcome [10].

When considering SE etiologies, it is helpful to keep in mind that the cause varies according to age group. In children, about $50 \%$ of SE occurs in the setting of infection with fever. Cerebrovascular disease (including acute and remote hemorrhagic and ischemic strokes) is a major cause of SE, accounting for about $40 \%$ of SE in adults and $60 \%$ of SE in old age $[1,11,12]$. Other common causes of SE in adults are low anti-epileptic drug (AED) level and remote symptomatic events $[6,7,12]$. In some locales, alcohol-related causes are a common etiology of SE $[7,12,13]$.

This study demonstrates that $84 \%$ of the SE patients showed abnormal ECG patterns within the $6 \mathrm{~h}$ following onset of SE. The most common changes were ischemia, arrhythmia and conduction defects. This suggests that significant risk factors for cardiac stress occur with SE. Our results are strikingly similar to those found in a study by Boggs et al in 1993 that documented ECG changes in a series of 60 patients and noted similar types of ischemic changes and arrhythmias (Table 1). The documentation of changing ECG tracings during SE and following the termination of SE indicates a dynamic, pathologic response of the myocardium to this neurological process [12].

The usual mortality rates in adult studies on SE range from $16 \%$ to $25 \%$, although risk steadily increases with advancing age. In the Greater Richmond Metropolitan Area Status Epilepticus Project (GRMASE) study, overall adult mortality was $26 \%$, but $38 \%$ for patients older than 60 years. This same study demonstrated SE-related mortality at greater than $50 \%$ in patients older than 80 years [14]. Etiology is probably the most obvious but complex determinant of SE mortality. Anoxia accounts for the highest mortality in older patients with SE, not unexpectedly after cardiac arrest. Overall, adult mortality from anoxia is $71 \%$ but reaches $92 \%$ in those patients older than 65 . These numbers are higher, but not significantly higher than mortality rates over 30 days af- 
Table 1. In a Review of the Literature, One Other Study by Boggs et al in 1993, Has Documented ECG Changes in a Series of 60 Patients. There Is a Striking Similarity to the Types of Changes Noted in These Studies, Particularly With Ischemic Changes and Arrhythmia.

\begin{tabular}{lll} 
EKG abn & $\begin{array}{l}\text { Our study } \\
(\mathbf{N = 1 1 3})\end{array}$ & $\begin{array}{l}\text { Boggs et al (1993) MCV } \\
(\mathbf{N = 6 0 )}\end{array}$ \\
\hline Ischemia & $39 \%$ & $40 \%$ \\
Conduction & $30 \%$ & $15 \%$ \\
Arrhythmia & $21 \%$ & $20 \%$ \\
Axis & $4 \%$ & $20 \%$ \\
Bradycardia & $3 \%$ & $3 \%$ \\
Voltage & $2 \%$ & $3 \%$ \\
\hline
\end{tabular}

ter in-hospital cardiac arrest in the absence of SE [14].

The overall mortality in our study was $7 \%(\mathrm{~N}=8)$. Of the eight patients who died, all of them had ECG changes while seven of these patients had persistent ECG changes. The mortality among patients with persistent ECG changes was $11 \%$ whereas $0.02 \%$ of patients with non-persistent ECG changes died. Our mortality rate is low is this study most likely due to the small number of patients who were experiencing new onset SE, which is associated with higher mortality and morbidity. Another factor contributing to our low rate of mortality could be improvements in medical management of patients with SE. The significant mortality associated with SE patients having persistent ECG abnormalities indicates that close observation of cardiac function is essential in managing $\mathrm{SE}$.

Boggs et al in 1993 found the mortality rate in patients with SE and ECG abnormalities to be $37 \%$ compared to patients with SE and no ECG abnormalities to be much lower (12\%) [12]. All eight patients who died in our study had ECG changes. The control of cardiovascular risk factors needs to be a part of the management of SE cases and can only be effected by thorough patient evaluation. With the advances made in parenteral pharmacotherapy, intensive care unit (ICU) management and the availability of high-quality EEG monitoring, one would expect that death rates from SE would have declined substantially in recent years; however, in order to decrease this number to its full potential, we must not just treat the emergency at hand. We must also treat the effects that SE has on other organs and the autonomic system in the hours and days following SE.

Changes to the brain caused by SE that lead to epileptogenesis have systemic effects, and alter cardiac activity [15]. The pathophysiology of ECG changes in SE is probably multifaceted. SE could cause cardiac arrhythmogenesis and depolarization-repolarization abnormalities through several mechanisms [16]. Mechanisms proposed for these abnormalities include excess release of catecholamines in the heart from sympathetic nerve terminals, increased circulating catecholamines due to stimulation of the adrenal medulla, or altered balance of autonomic outflow due to specific neurologic lesions of hypothalamus, limbic cortex, reticular formation and stellate ganglia [16]. SE may also cause ECG changes by causing severe physiologic stress with resultant sympathetic over- activity, metabolic acidosis and decompensation of underlying myocardial compromise [17]. The pathological profile has been called "catecholamine cardiomyopathy" and has been found in patients with acute stress response to trauma and in some patients with sudden death [18]. The true incidence of such lesions in acute and chronic illness of various types has not been investigated systematically, but may be an underlying etiology in sudden death [12].

In the presence of occult coronary artery disease, convulsive SE may act as a "physiologic stress test", by increasing myocardial oxygen demand and inducing gross and microscopic damages to the heart. In cases of non-convulsive $\mathrm{SE}$, it is difficult to hypothesize the mechanical sources of ECG abnormalities. Some of the ECG changes seen in these patients may be a functional manifestation of microscopic myocardial abnormalities, which have been implicated in sudden death. Similar mechanisms of microscopic damage may significantly contribute to high mortality of SE [12].

The majority of ECG abnormalities seen in our patients with SE resolved spontaneously; however, potentially life threatening ECG abnormalities that did not resolve within a reasonable length of time were treated and reversed with medications and pacemakers. Ten patients developed elevated cardiac enzymes and ECG changes less than $6 \mathrm{~h}$ from SE onset consistent with myocardial infarction (9\%). Three of these patients died, two of which were experiencing new on- 
set epilepsy. The majority of patients with new onset epilepsy who died developed ECG abnormalities which persisted. These abnormalities include ST elevation, bradycardia and prolonged Q-T interval. It is yet to be determined if the type of ECG abnormality recorded during SE is as significant as the lability of the ECG seen during and after SE [12] and due to our small sample size we are unable to decipher which is more predictive of death. Our results indicate that ischemic changes, ST depression or elevation, and PVCs were the most common ECG abnormalities recorded in patients who died. However, our sample of patients who died is small, and bradycardia, prolonged QT and RBBB closely followed as arrythmogenic changes preceding death.

Guidelines for the evaluation and management of status epilepticus (2012) recommend a stepwise approach to management of SE; however, they acknowledge the lack of scientific evidence needed to create a more comprehensive evidence-based approach to the care of these patients [5]. This can only be determined by systematically examining outcomes resulting from national clinical pathways and randomized controlled trials [5]. It has been proven that $\mathrm{SE}$ causes intense activation of the sympathetic nervous system and results in myocyte myofilament damage, arrhythmogenic alterations in cardiac electrical activity and increased susceptibility to ventricular arrhythmias [19, 20], yet the prognostic value of these cardiac changes has yet to be determined.

This study provides great insight into ECG use as a painless and cost effective prognostic tool in the monitoring and treatment of SE. Knowing that persistent ECG changes in the hours and days following SE are predictive of increased morbidity and mortality gives us the information we need to begin to change the way we manage and monitor SE patients. Our results indicate that cardiac monitoring needs to be a part of the evaluation and treatment of SE to allow early detection of potentially life threatening cardiovascular abnormalities.

Further studies must be done to determine how much early detection of cardiovascular abnormalities will change the prognosis of a patient who undergoes SE with subsequent ECG changes. This will only be possible to determine once ECG monitoring is conducted in a large number of patients with SE.

\section{Grant Support}

This research received no specific grant from any funding agency in the public, commercial, or not-for-profit sectors.

\section{Disclosure Statement}

The authors declare that they have no competing interests.

\section{Abbreviations}

SE: status epilepticus; ECG: electrocardiogram; PVC: premature ventricular contraction; Afib: atrial fibrillation; Aflu: atrial flutter; PAC: premature atrial contraction; Vfib: ventricular fibrillation; LBBB: left bundle branch block; RBBB: right bundle branch block; LAFB: left anterior fascicular block; IVCD: intra-ventricular conduction delay; AVB: AV block; AED: anti-epileptic drugs; DAED: discontinued antiepileptic drugs; GRMASE study: Greater Richmond Metropolitan Area Status Epilepticus Project.

\section{References}

1. DeLorenzo RJ. Status epilepticus: concepts in diagnosis and treatment. Semin Neurol. 1990;10(4):396-405.

2. Maytal J, Shinnar S, Moshe SL, Alvarez LA. Low morbidity and mortality of status epilepticus in children. Pediatrics. 1989;83(3):323-331.

3. Hauser WA. Status epilepticus: epidemiologic considerations. Neurology. 1990;40(5 Suppl 2):9-13.

4. Rossetti AO, Lowenstein DH. Management of refractory status epilepticus in adults: still more questions than answers. Lancet Neurol. 2011;10(10):922-930.

5. Brophy GM, Bell R, Claassen J, Alldredge B, Bleck TP, Glauser T, Laroche SM, et al. Guidelines for the evaluation and management of status epilepticus. Neurocrit Care. 2012;17(1):3-23.

6. Aminoff MJ, Simon RP. Status epilepticus. Causes, clinical features and consequences in 98 patients. Am J Med. 1980;69(5):657-666.

7. DeLorenzo RJ, Towne AR, Pellock JM, Ko D. Status epilepticus in children, adults, and the elderly. Epilepsia. 1992;33(Suppl 4):S15-25.

8. Fisch C. Abnormal ECG in clinically normal individuals. JAMA. 1983;250(10):1321-1323.

9. DeLorenzo RJ, Pellock JM, Towne AR, Boggs JG. Epidemiology of status epilepticus. J Clin Neurophysiol. 1995;12(4):316-325.

10. Treatment of convulsive status epilepticus. Recommendations of the Epilepsy Foundation of America's Working Group on Status Epilepticus. JAMA. 1993;270(7):854859.

11. Gilchrist JM. Arrhythmogenic seizures: diagnosis by simultaneous EEG/ECG recording. Neurology. 1985;35(10):1503-1506.

12. Boggs JG, Painter JA, DeLorenzo RJ. Analysis of electrocardiographic changes in status epilepticus. Epilepsy Res. 1993;14(1):87-94.

13. Haft JI. Cardiovascular injury induced by sympathetic catecholamines. Prog Cardiovasc Dis. 1974;17(1):7386.

14. Boggs JG. Mortality Associated with Status Epilepticus. 
Epilepsy Curr. 2004;4(1):25-27.

15. Kheiri F, Bragin A, Engel J, Jr., Almajano J, Winden E. Non-linear classification of heart rate parameters as a biomarker for epileptogenesis. Epilepsy Res. 2012;100(1-2):59-66.

16. Samuels MA. Neurogenic heart disease: a unifying hypothesis. Am J Cardiol. 1987;60(18):15J-19J.

17. Stenstrom G, Swedberg K. QRS amplitudes, QTc intervals and ECG abnormalities in pheochromocytoma patients before, during and after treatment. Acta Med Scand. 1988;224(3):231-235.
18. Baroldi G. Different morphological types of myocardial cell death in man. Recent Adv Stud Cardiac Struct Metab. 1975;6:383-397.

19. Mehrpour M, Hajsadeghi S, Fereshtehnejad SM, Bassir P. Serum levels of cardiac troponin I in patients with status epilepticus and healthy cardiovascular system. Arch Med Res. 2013;44(6):449-453.

20. Bealer SL, Little JG, Metcalf CS, Brewster AL, Anderson AE. Autonomic and cellular mechanisms mediating detrimental cardiac effects of status epilepticus. Epilepsy Res. 2010;91(1):66-73. 